\title{
Destruction of a spherical polystyrene microparticle in pulsed ultraviolet beam for micromanipulation purpose
}

\author{
Roman V. Skidanov, and Vadim S. Vasiliev* \\ Department of Technical Cybernetics, Samara State Aerospace University, 151 Molodogvardeyskaya Str., Samara \\ 443086, Russia \\ *e-mail: vadimka.vasilev.93@mail.ru
}

\begin{abstract}
The modelling of heating a spherical polystyrene microparticle by the laser beam having the wavelength $355 \mathrm{~nm}$ and varying spot size is described. The simulation results are presented for two different values of the beam spot radius. Nature experiments are reported aimed at measuring the velocity of a particle with the radius $5 \mu \mathrm{m}$ accelerated by microexplosion of the neighbouring particle. (C) 2016 Journal of Biomedical Photonics \& Engineering.
\end{abstract}

Keywords: microexplosion, ultraviolet light beam, polystyrene microspheres, microparticle velocity.

Paper \#2995 received 2016.03.11 revised manuscript received 2016.03.24; accepted for publication 2016.03.24; published online 2016.03.31. doi: 10.18287/JBPE16.02.010306

\section{References}

1. P. Zemánek, A. Jonáš, L. Šrámek, and M. Liška, “Optical trapping of nanoparticles and microparticles by a Gaussian standing wave,” Opt. Lett. 24(21), 1448 (1999).

2. A. K. De, D. Roy, A. Dutta, and D. Goswami, "Stable optical trapping of latex nanoparticles with ultrashort pulsed illumination," Applied Optics 48(31), G33 (2009).

3. L. Bosanac, T. Aabo, P. M. Bendix, and L.B. Oddershede, "Efficient Optical Trapping and Visualization of Silver Nanoparticles," Nano Letters 8(5), 1486-1491 (2008).

4. G. Thalhammer, R. Steiger, M. Meinschad, M. Hill, S. Bernet, and M. Ritsch-Marte, "Combined acoustic and optical trapping," Biomed. Opt. Express 2(10), 2859 (2011).

5. R. V. Skidanov, A. A. Morozov, and A. P. Porfirev, "Composite light beam and microexplosion for optical micromanipulation,” Kompyuternaya optika 36(3), 371-376 (2012) [in Russian].

\section{Introduction}

At present, all studies on manipulation are progressing towards the reduction of the moved object size. [1-3]. A variety of methods for manipulating microparticles using optical traps of different types exists. However, it is desirable to have a method for moving relatively large microscopic objects (larger than $30 \mu \mathrm{m}$ ). Such objects most frequently observed in biological studies (spores, tissue microsamples, large cells). In this case, it is desirable to prevent the light incidence on the microscopic object. Mechanical manipulations can be implemented using mechanical tweezers; however, it can damage the moved object.

The characteristic size of the objects moved by a common optical trap varies from parts of a micrometre to nearly ten micrometres. With the increase of the object size, an essential increase of the light beam power is necessary. Due to the absorption of the light beam energy, the maximal size exists for the microparticle that can be moved at the expense of optical capture forces. The exact value of this size depends on many parameters, e.g., the absorption coefficient of the microscopic object, the properties of the liquid, the surface shape of the object, etc. Approximate estimation of this size yields about $30 \mu \mathrm{m}$ for spherical transparent microscopic objects. It is worth noting that the object with the size close to this limit value experiences extreme thermal load. Even worse is the situation for manipulating with non-transparent objects by means of light traps. In this case, the limiting size is reduced by 1.5-2 times. A rather sophisticated method of combined trapping exists for such microscopic objects [4], e.g., using light and ultrasound. In Ref. [5] a simpler method of driving such 
microobjects is described, basing on microexplosions of polystyrene microparticles in the ultraviolet laser beam with the wavelength $355 \mathrm{~nm}$.

Using this method it is also possible to move sufficiently large microobjects (including the biological ones) without loss or partial destruction of the manipulated object. Using the microexplosion one can implement the displacement of the adjacent object with definite direction and distance.

The subject of the present study are the motion parameters of microobjects driven by the explosion of a polystyrene microparticle.

The goal of this works was to calculate, simulate and experimentally verify the parameters at which the spherical polystyrene particle explodes in the pulsed ultraviolet laser beam.

In correspondence with the goal, the following problems were formulated:

- development of the method for calculating the parameters, at which the microparticle explosion occurs, such as the microparticle temperature and the minimal power, providing the explosion;

- conduction of natural experiment on the microparticle explosion and qualitative comparison of the results with those obtained by simulation;

- calculation of particle dispersion velocity for different focusing objectives $(20 \times, 40 \times, 100 \times)$.

\section{Mathematical model describing the heating of a polystyrene particle}

The heating of a polystyrene particle under the effect of pulsed ultraviolet laser radiation can be described by the heat conduction equation with the boundary conditions:

$$
\left\{\begin{array}{l}
\frac{\partial u}{\partial t}-a^{2} \Delta u=f(r, t) \\
\left.u\right|_{t=0}=T_{0} \\
\left.u\right|_{r=R}=T_{0},
\end{array}\right.
$$

We additionally consider the equation for the initial layer $r_{0}$ having the form

$$
u_{1}=\frac{P}{c m} \frac{\left(r_{1}-r_{0}\right)}{r_{1}}+\left.u\right|_{t=0} \text {. }
$$

For the convenience of numerical solution let us consider the two-dimensional case with the nodes of the coordinate grid $\left(r_{i}, t_{k}\right)$. After the discretisation of each component in Eq. (1), transformation to cylindrical coordinates and taking the spherical symmetry of the problem into account (all heat is accumulated in the centre and propagates in all directions uniformly) we arrive at the following formula:

$$
\begin{aligned}
& \frac{u_{i}^{k+1}-u_{i}^{k}}{h_{t}}-a^{2}\left(\frac{u_{i+1}^{k+1}-2 u_{i}^{k+1}+u_{i-1}^{k+1}}{h_{r}^{2}}+\right. \\
& \left.+\frac{2}{r_{i}} \frac{u_{i+1}^{k+1}-u_{i}^{k+1}}{h_{r}}\right)=\frac{P}{c m}
\end{aligned}
$$

where $P$ is the power of laser radiation, $c$ is the specific heat capacity of the polystyrene particle.

\section{Results of modelling}

We present the results of the modelling carried out with different parameters of laser impact. The results of the modelling are presented in Figs. 1 and 2.

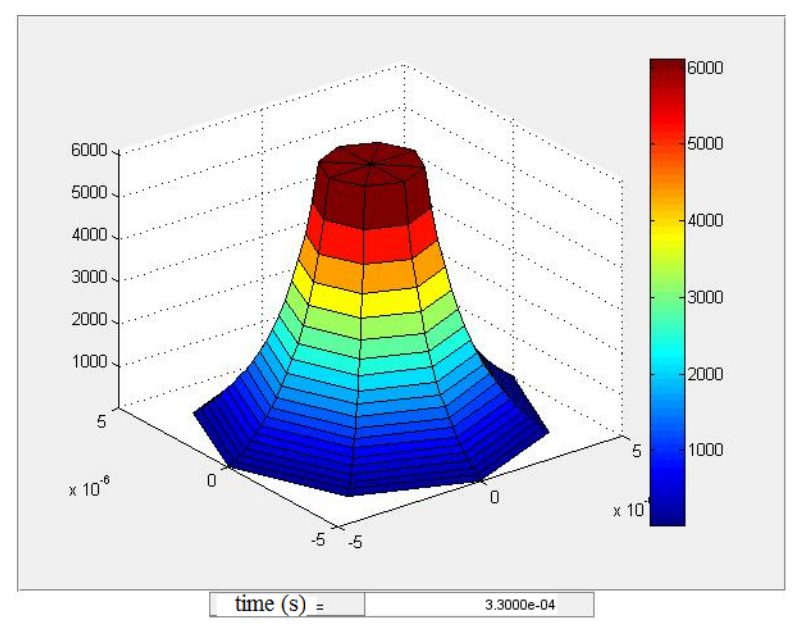

Fig. 1 Modelling of the polystyrene particle heating process with the parameters used in the experiment.

Figure 1 shows the results of modelling with the following parameters: the laser radiation power 15.4 $\mathrm{mW}$, the pulse repetition rate $3000 \mathrm{~Hz}$, which corresponds to the period of $0.33 \cdot 10^{-3} \mathrm{~s}$. The diameter of the polystyrene particle was $5 \mu \mathrm{m}$ and the laser beam spot diameter at the particle surface was $1.5 \mu \mathrm{m}$. These parameters correspond to the experimental ones. One can see that in this case the maximal temperature amounts to $6114 \mathrm{~K}$. This is much higher than the temperature of polystyrene destruction. This model is correct only below a certain temperature, because the base material of the microobject becomes modified. However, the model demonstrates that such critical parameters are achieved in a very short time, i.e., one pulse with the duration $5 \mathrm{~ns}$ is sufficient for complete explosive destruction of the microparticle, which will be experimentally confirmed below.

Let us see what happens if the diameter of the beam spot at the particle surface increases to $3 \mu \mathrm{m}$, which corresponds to using the focusing objective $40 \times$. This situation is illustrated in Fig. 2. All other parameters are the same as in Fig. 1. 


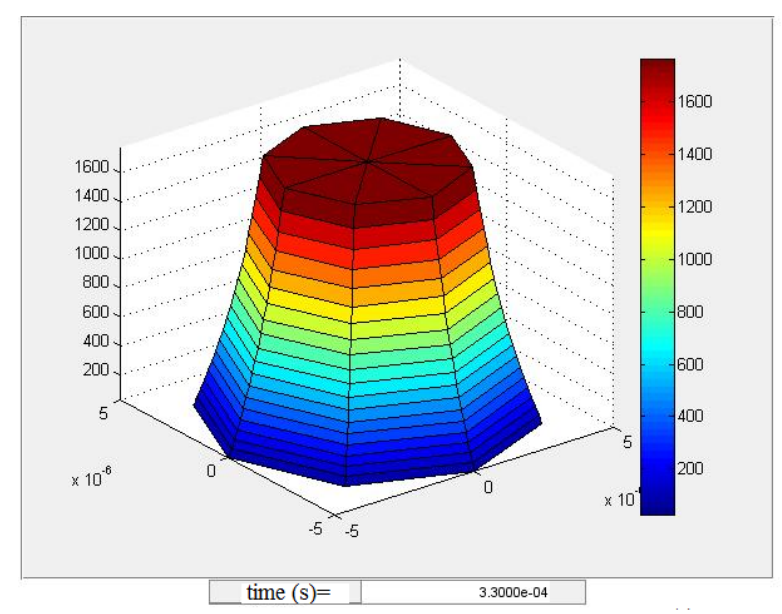

Fig. 2 Modelling of the polystyrene particle heating process with the parameters used in the experiment and the laser spot diameter of the particle surface equal to 3 $\mu \mathrm{m}$.

Now the maximal value of the temperature appears to be $1761 \mathrm{~K}$. As seen from the simulation results, during the pulse with the energy about $5.4 \mu \mathrm{J}$ the polystyrene microparticles is also heated to significant temperatures that exceed the temperature of polystyrene destruction. Since the model allows only the estimation of the heating parameters below the temperature of polystyrene destruction, the quantitative estimation of the microexplosion parameters requires natural experimental study.

\section{Experiment}

The schematic diagram of the experimental setup is presented in Fig 3. The setup incorporates the ultraviolet (UV) laser DTL-375 with the wavelength $355 \mathrm{~nm}$ and the maximal mean power $40 \mathrm{~mW}$ (the maximal pulse energy $5.6 \mu \mathrm{J}$, the pulse duration $10^{-9} \mathrm{~s}$, the pulse repetition rate $3000 \mathrm{~Hz}$ ), the rotary mirrors $\mathrm{M}_{1}, \mathrm{M}_{2}, \mathrm{M}_{3}$; the illumination lamp $\mathrm{I}$, the focusing microscope objective $L_{1}(20 \times, 40 \times, 90 \times)$; the imaging microscope objective $L_{2}(16 \times)$; the CCD camera and the substrate with microobjects $\mathrm{V}$. In the experiment, we used the DDC camera FastVideo $500 \mathrm{E}$ with the resolution $640 \times 480$.

The substrate was made of silica glass. This material does not absorb light in the UV range, thus excluding the microexplosion due to the heating and melting of the glass surface. Below we present the experimental results on the microparticle explosion far from the surface of the silica substrate.

Using multiple recorded frames, we calculated the mean values of the velocity of the polystyrene particles pushed away by the explosion of the neighbouring polystyrene particle with the diameter $5 \mu \mathrm{m}$.

The calculations were carried out with 3 different focusing microscope objectives $(20 \times, 40 \times, 100 \times)$ to determine the optimal parameters of focusing.

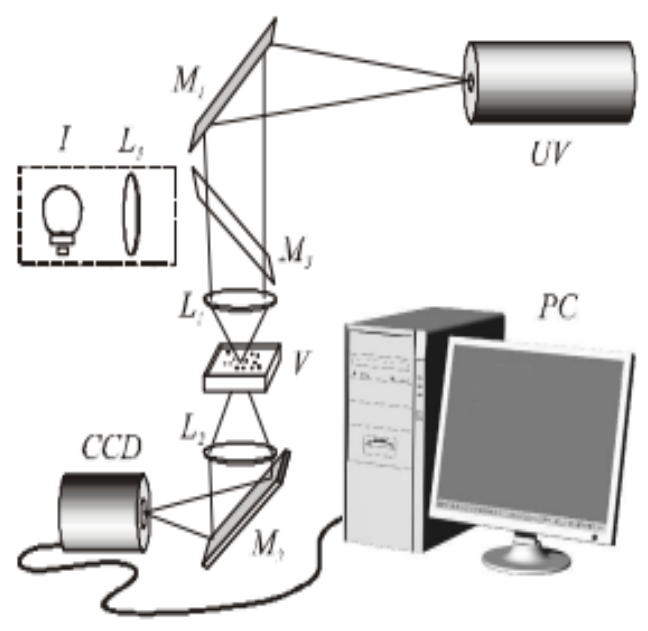

Fig. 3 Optical scheme of the experimental setup for investigating the explosion of polystyrene microparticles.

For the focusing objective $20 \times$ the beam diameter amounted to $8 \mu \mathrm{m}$, and the diameter of the spot on the particle was $4-5 \mu \mathrm{m}$. The mean velocity of spreading polystyrene particles amounted to $15.53 \mathrm{~mm} / \mathrm{s}$.

For the focusing objective $40 \times$ the beam diameter amounted to $4 \mu \mathrm{m}$, and the diameter of the spot on the particle was $2-3 \mu \mathrm{m}$. The mean velocity of spreading polystyrene particles amounted to $10.53 \mathrm{~mm} / \mathrm{s}$.

When using the focusing objective $100 \times$ the beam diameter amounted to $1.5 \mu \mathrm{m}$, and the diameter of the spot on the particle was $1 \mu \mathrm{m}$. The mean velocity of spreading polystyrene particles amounted to $9.61 \mathrm{~mm} / \mathrm{s}$. As seen from the results, the increase of the objective focal power did not increase the energetics of microparticle motion. This fact is probably due to large losses of light in the objectives with high magnification.

\section{Conclusion}

In the course of performing the present work, the following results were obtained:

- The model of heating a spherical polystyrene microparticle with the laser beam focused at the microparticle centre was developed. The results of modelling at different parameters were obtained.

- The velocities of particles spreading by the microexplosion were experimentally determined using different focusing objectives. For the objectives $20 \times$, $40 \times$, and $100 \times$ the mean velocity amounted to 15.3 , 10.53 , and $9.61 \mathrm{~mm} / \mathrm{s}$. The decrease of the particle velocity is obviously due to the loss of light in microscope objectives, which is essentially higher in the objectives with higher magnification. 

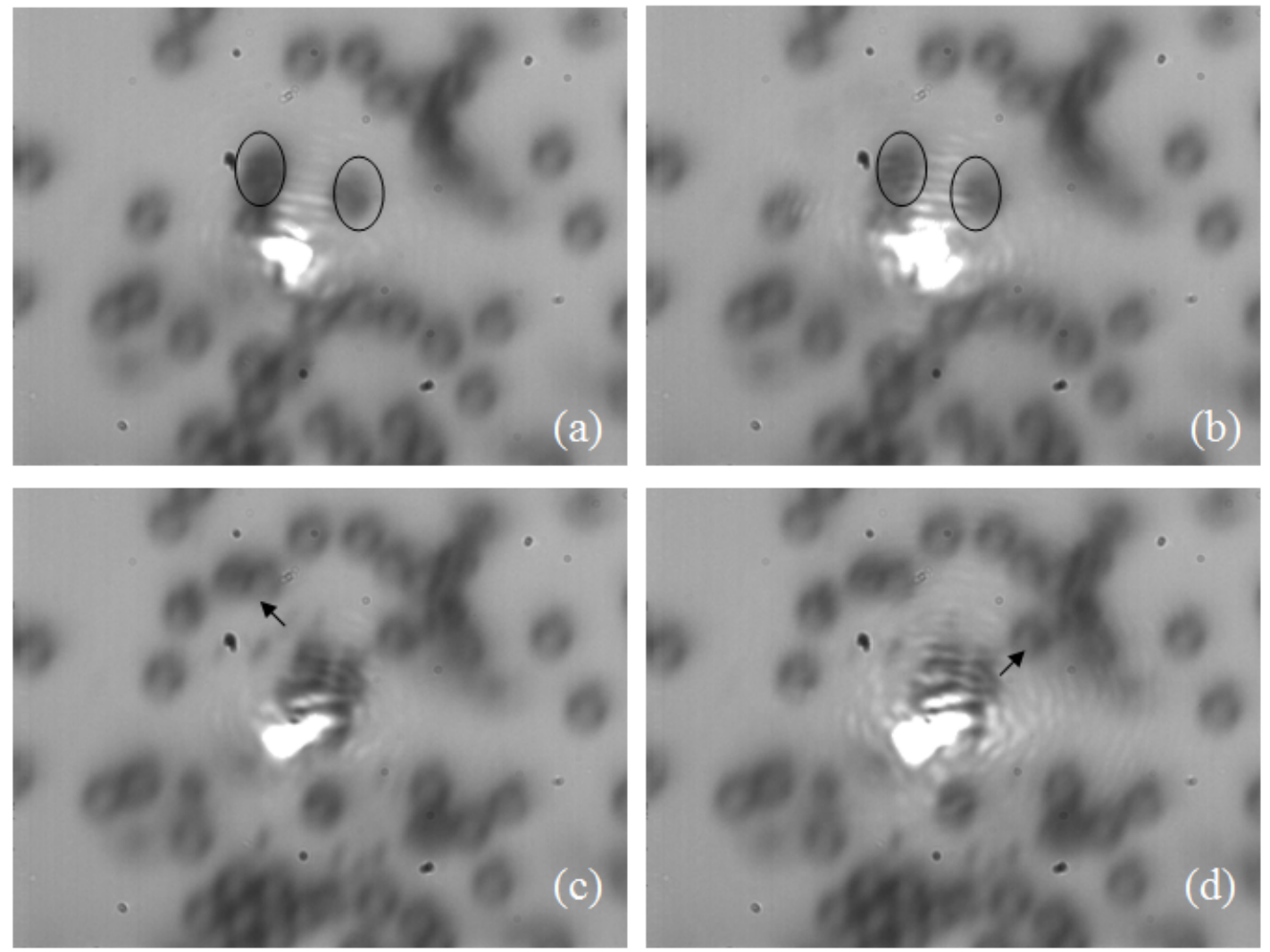

Fig. 4 Displacement of polystyrene microparticles due to the central particle explosion, recorded with the time interval $0.525 \mathrm{~ms}$, the image scale being $1 \mu \mathrm{m}$ in $1 \mathrm{~mm}$. 